\title{
Phonon Reflections from Nanostructured Interfaces Imaged by Momentum- Averaged and Resolved Vibrational EELS
}

\author{
Chaitanya Gadre ${ }^{1}$, Xingxu Yan ${ }^{2}$, Qichen Song ${ }^{3}$, Gang Chen ${ }^{3}$ and Xiaoqing Pan $^{1}$
}

${ }^{1}$ Department of Physics and Astronomy, University of California, Irvine, CA 92697, Irvine, California, United States, ${ }^{2}$ Department of Materials Science and Engineering, University of California - Irvine, Irvine, California, United States, ${ }^{3}$ Michigan Institute of Technology, United States

Phonon transport is the dominant factor in determining thermal conductivity in semiconductors and is greatly modulated by nanostructures, interfaces, and defects but the physics of the nanoscale process has hardly been explored by experimental means. The phonon resistance provided by these structures are often a result of a mismatch between the local phonon density of states (LDOS) [1], studies of which have eluded optical measurement techniques due to their insufficient spatial resolution. Additionally, optical, and other conventional phonon spectroscopies have no way of investigating the phonon dynamics of individual interfaces and as a result, cannot aid in the physical understanding of nanoscale thermal transport. However, recent developments in electron microscopy have enabled the acquisition of vibrational spectra at sub-nanometer resolutions via monochromated electron energy loss spectroscopy (EELS) [2]. Armed with this technical capability, we demonstrate a mapping of phonons revealing interface dynamics at the nanometer scale. Utilizing momentum- averaged and resolved EELS, we image phonon reflections from nanostructure interfaces revealing the dynamics of phonon propagation and reflection.

We employ a momentum averaged beam scattering geometry depicted in Fig. 1A to study the intensity of the Si optical mode (OM). Fig. 1B shows a high angle annular dark field (HAADF) image of the left side of a QD consisting of a gradual Si-SiGe at the top and a more abrupt SiGe-Si interface at the bottom elucidated by the composition gradient map in Fig. 1C. Fig. 1D is a mapping of the Si OM revealing an abnormal intensity enhancement in the presence of the two interfaces. It can be seen from the profile in Fig. 1E, the region towards the center of the QD, that the Si OM mode intensity is significantly greater than that at the top of the QD while at the edge of the QD (Fig. 1F) that the intensity in the vertical direction is far more uniform. The compositional gradient plots in Figs. 1E-1F suggest that the sharpness of the interfaces is modulating this intensity.

To see propagating modes, a 3 mrad convergence semi-angle probe was used to enable the investigation of phonon modes at specific points in $k$-space within the first Brillouin zone (FBZ) (Fig. 2A). By using post-specimen lens deflectors, the scattered off-axis beam was collected at points $\Delta_{+}$and $\Delta_{\text {- denoted in }}$ Fig. 2B. By conservation of energy and electron-phonon momentum, these points in momentum space within FBZ correspond to downward and upward propagating phonon modes, respectively. The difference then provides net momentum information i.e. direction of propagation. Utilizing this novel technique, the differential momentum mapping in fig. $2 \mathrm{C}$ reveals that there is indeed a strong reflection from the abrupt interface while the gradual interface provides a weaker directionality. The abrupt change in LDOS creates an environment where propagating Si optical modes experience a shortage of corresponding modes on the other side of the interface, leading to a reflection.

Our work showcases the versatility of vibrational EELS for studying nanoscale physics previously invisible to experimental techniques. By utilizing averaged and resolved momentum conditions, phonon 
momenta can be imaged to obtain information about phonon propagation at the nanometer scale. We have shown direct evidence of phonon reflection from interfaces of differing specularity. These ideas and techniques can be extended for investigating phonon dynamics of nanostructures, interfaces, and defects in the presence of thermal gradients and free charge carriers.
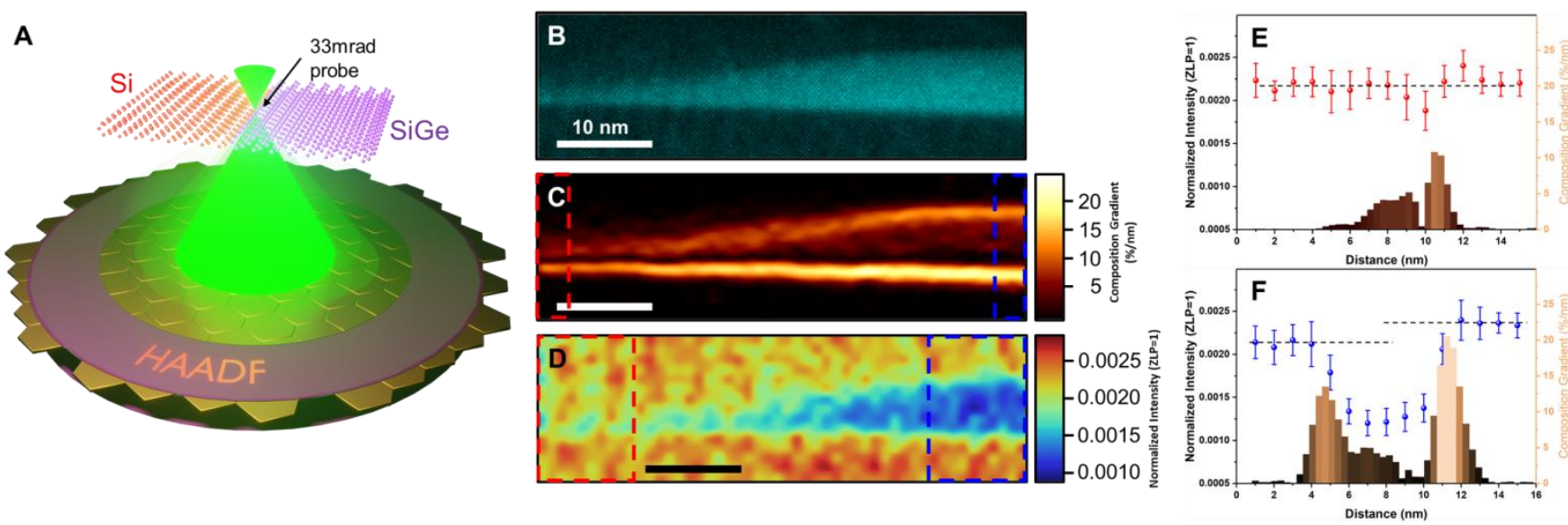

Figure 1. Figure 1: Momentum Averaged Si OM Intensity Mapping of a partial QD. (A) Schematic of momentum averaged beam scattering geometry. A 33mrad probe covers multiple BZ's. (B) HAADF image of left half of a QD. (C) Vertical gradient of Ge composition of QD in A. (D) Si OM intensity map of QD in A. Scale bars in B-D are 10nm. (E,F) Horizontally averaged line profile of Si optical mode intensity over the region in D marked in red and blue, respectively. 1D profiles of the composition gradient in $\mathrm{C}$ marked in red and blue, respectively. The intensity enhancement is nearly absent where the interfaces converge (red).
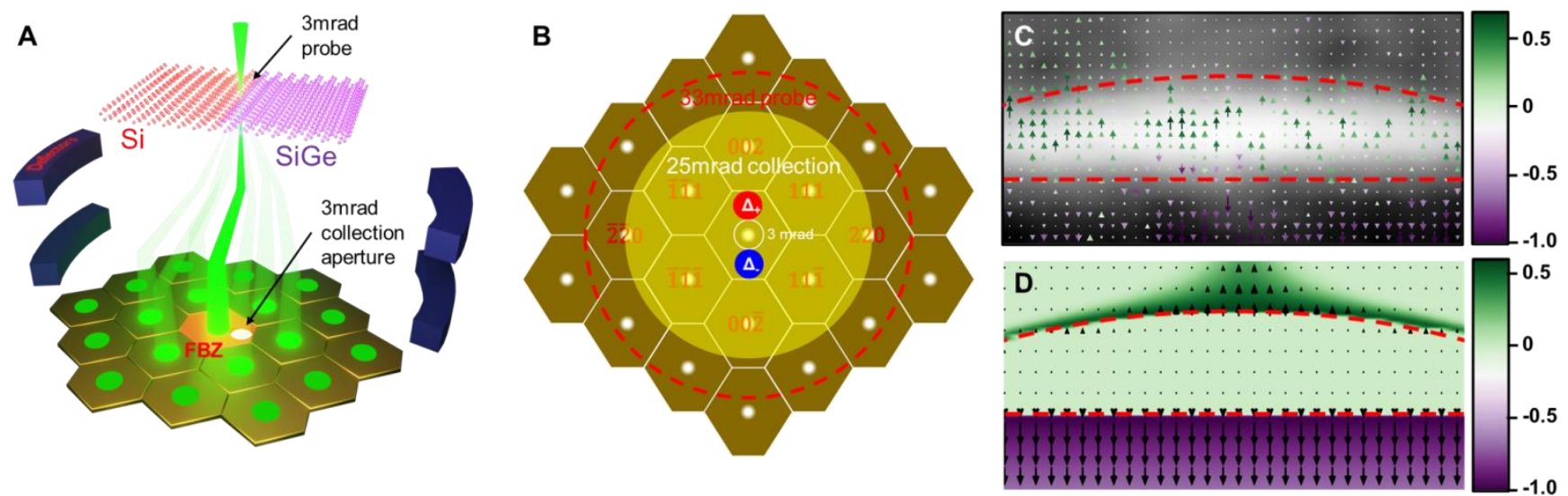

Figure 2. Figure 2: Momentum resolved differential mapping of phonon reflection. (A) Momentum resolved beam-scattering geometry. Post specimen deflectors tilt the beam so that the off-axis signal enters the 3mrad EELS collection aperture. A 3mrad convergence semi-angle probe is about $1 / 3$ the size of the FBZ in Si. (B) Reciprocal space schematic diagram of Si. White borders denote individual Brillion zones (BZs). Various beam-detector geometries corresponding to momentum-averaged and momentumresolved conditions are denoted by circles with their size in semi-angle labeled. Red and blue regions in the FBZ correspond to the areas where momentum resolved differential data was obtained. (C) Phonon reflections obtained from differentially mapped phonons. (D) BTE simulation showing asymmetric 
reflection of phonons from gradual (top) and abrupt (bottom) interfaces. The magnitude of reflections from the abrupt interface in $\mathrm{B}$ and $\mathrm{C}$ is greater than the gradual one due to increased interface specularity.

References

[1] Swartz, Eric T., and Robert O. Pohl. "Thermal boundary resistance."Reviews of modern physics" 61.3 (1989): 605.

[2] Krivanek, Ondrej L., et al. "Vibrational spectroscopy in the electron microscope." Nature 514.7521 (2014): 209.

[3] This work was supported by the Department of Energy (DOE) under Grant DE-SC0014430. The authors acknowledge the use of facilities and instrumentation at the UC Irvine Materials Research Institute (IMRI) supported in part by the NSF MRSEC program (DMR-2011967). 